\title{
Mitteilungen der ÖGIAIN
}

Med Klin Intensivmed Notfmed 2012 .

107:230-230

DOI 10.1007/s00063-012-0105-2

(C) Springer-Verlag 2012

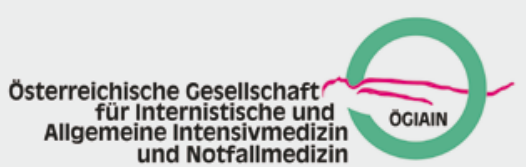

Österreichische Gesellschaft für Internistische und Allgemeine Intensivmedizin und Notfallmedizin (ÖGIAIN)

\section{Sekretär}

Univ.-Prof. Dr. S. Dunzendorfer

Medizinische Universität Innsbruck

Anichstraße 35, 6020 Innsbruck

Tel. +43 (512) 504-81302

Fax +43 (512) 504-24199

Stefan.Dunzendorfer@i-med.ac.at

www.intensivmedizin.at

\section{Namensänderung der ÖGIAIM}

\begin{abstract}
Anlässlich der Wiener Intensivmedizinischen Tage (WIT) 2012 wurde auf Antrag des Vorstandes der Gesellschaft eine außerordentliche Generalversammlung einberufen und am 16.2.2012 die Namensänderung auf Österreichische Gesellschaft für Internistische und Allgemeine Intensivmedizin und Notfallmedizin (ÖGIAIN)

mehrheitlich beschlossen. Subsequent soll innerhalb der ÖGIAIN auch eine Arbeitsgruppe für Notfallmedizin eingerichtet werden.
\end{abstract}

Die Umbenennung der Gesellschaft erfolgte auf der Basis folgender Überlegungen:

1. Im klinischen Alltag kommt es regelmäßig präklinisch als auch innerklinisch zu einer

Überschneidung internistisch notfallmedizinischer und internistisch intensivmedizinischer diagnostischer und therapeutischer Aufgabenstellungen und Handlungen. Dieser Situation soll die Umbenennung in ÖGIAIN und die dementsprechende Spezifizierung der Agenden der Gesellschaft in den Satzungen gerecht werden.

2. Die Fortbildungsveranstaltungen der Gesellschaft decken schon seit Jahren nicht nur intensivmedizinische sondern auch notfallmedizinische Themen ab.
3. Die Fachkompetenz und ärztliche Tätigkeit der Mehrheit unserer Mitglieder beschränkt sich nicht auf internistische Intensivmedizin sondern beinhaltet zu einem sehr großen Anteil auch internistische Notfallmedizin.

4. Die wichtigsten Printmedien der ÖGIAIN publizieren mit einem signifikanten Anteil auch internistische notfallmedizinische Artikel (Medizinische Klinik - Intensivmedizin und Notfallmedizin bzw. INTENSIV-News - Forum für Intensiv- und Notfallmedizin).

Aus den genannten Gründen konnte eine offizielle Erweiterung der Agenden und damit die notwendige Umbenennung der Gesellschaft nur begrüßt werden.

Die aktuellen Veranstaltungen der ÖGIAIN entnehmen Sie bitte unserer bereits aktualisierten homepage: www.intensivmedizin.at.

Univ.-Prof. Dr. S. Dunzendorfer Wissenschaftl. Sekretär der ÖGIAIN 